\title{
Balking and Reneging in the Queuing System
}

\author{
Dr.Mamata Kuila \\ Department of Mathematics, College of Basic Science \& Humanities, \\ Orissa University of Agriculture \&Technology, Bhubaneswar, Odisha, India-751003
}

\begin{abstract}
In this paper, we have discussed about a steady state solution of the ordered queuing problem with balking and reneging. Here we have taken the waiting line is of chi-square queue with Poisson balking probability which depend not only on the number of customers in the system, but also the rate of services in the system.
\end{abstract}

Keywords: Balk Queue, Renege, Rate of Service, Steady state.

\section{Introduction}

A queue, or a waiting line, involves arriving items that wait to be served at the facility which provides the service they seek. Queuing theory is concerned with the statistical description of the behavior of the queues with finding, e.g., the probability distribution of the number in the queue from which the mean and variance of queue length and the probability distribution of waiting time for a customer, or the distribution of a server's busy period can be found. In this paper we have discussed about a steady state solution of the ordered queuing problem with balking and reneging. In the case of balking, immediately on arrival a unit decides not to join the queue, perhaps because of the length of the queue or because of other information on the length of service. A unit reneges (i.e., becomes impatient and leaves without having been served) after joining the queue if it is decided that the wait will be longer than can be tolerated. Here the waiting line is of Chi-square queue with Poisson balking probability which depend not only on the number of customers in the system, but also on the rate of service in the system.

A queuing situation with the following characteristics has been considered.

i) An arriving customer may not join the queue if there are many customers in the system i.e., the customer may balk.

ii) A customer receives the service immediately, when the system is empty .But a joining customer that has to wait for service due to impatience may leave i.e., the customer may renege.

iii) Service is performed on the customer at the head of the line.

iv) One service has commenced on a customer ,it remains until the completion of service.

Barrer [1] has studied the problem of a unit leaving a queue after having waited longer than an acceptable time. O.Brien[4] has also found the solution of some queuing problem .Miller [3 ] and Konigsberg [2] have also studied about balk queue and queuing with special service. In this paper we have attempted to find out a steady state solution of chi - square queue, when the poison probabilities depend not only on the number of the customers in the system.

\section{Assumptions}

a) Customers arrive from a single infinite source with inter arrival time density function

$$
\begin{aligned}
a(t) & =\frac{1}{\Gamma\left(\frac{n}{2}\right) 2^{n} / 2} t^{\frac{n}{2}-1} e^{-\frac{t}{2}} \\
& =f_{k}(\lambda) t^{k-1} e^{-k \lambda t}
\end{aligned}
$$

Where $f_{k}(\lambda)=\frac{(k \lambda)^{k}}{(k-1) !}, k \geq 1,0 \leq t<\infty$ and $\lambda$ is the mean arrival.

b)An arriving customer joins the system with probability $e_{n}=e^{-n \alpha / \mu},(0 \leq n<\infty)$,

where $\alpha$ is a measure of customer's willingness to join the queue, $\mathrm{n}$ is the number in the system Including the one in service facility and $\mu$ is the rate of service.

c)After joining the queue, each customer will wait a certain length of time for service to begin. If it has not begun by then, he departs. This time is a random variable whose density function is

$$
\begin{gathered}
C(t)=f_{k}(\beta) t^{k-1} e^{-k \beta t} \\
, k \geq 1,0 \leq t<\infty
\end{gathered}
$$

d) Customers are served in order of arrival(FCFS) model by a single service facility. Once service commences, it always proceeds to completion. The service density function is

$$
S(t)=f_{k}(\mu) t^{k-1} e^{-k \mu t} \quad 0 \leq t<\infty, k \geq 1
$$




\section{The State Probabilities}

Since any one of the (n-1) customers in the queue may renege, we need the joint distribution for minimum of (n-1) selection. The corresponding density function $C_{n-1}(t)$ can be found out. we have $C(t)=f_{k}(\beta) t^{k-1} e^{-A t} \quad$, where $k \beta=A$

Hence $C_{n}(t)=f_{k}(n \beta) t^{k-1} e^{-n A t}$

If $P_{n}(t)$ is the transient probability of $\mathrm{n}$ units in the queue at time $\mathrm{t}$ and if $C_{n}\left(\tau_{0}, t\right) \Delta t+o(\Delta t)$ is the conditional probability that a unit reneges in $(t, t+\Delta t)$ if the queue length is $\mathrm{n}$ at time $\mathrm{t}$ and its fixed waiting limit is $\tau_{0}$, then we have

$p_{0}^{\prime}(t)=-\lambda p_{0}(t)+\left[\mu+C_{1}\left(\tau_{0}, t\right)\right] p_{1}(t)$

$p_{n}^{\prime}(t)=\lambda e_{n-1} p_{n-1}(t)-\left[\lambda e_{n}+\mu+C_{n}\left(\tau_{0}, t\right)\right] p_{n}(t)+\left[\mu+C_{n+1}\left(\tau_{0}, t\right)\right] p_{n+1}(t), n \geq 1$

If $p_{n}$ is the steady state probability of $n$ customers in the stream, and if $r=e^{-a / 2}$ conditional probability that a unit reneges in time $(t+\Delta t)$, if the queue length is $n$ at time $t$ and its fixed waiting limit is $\tau_{0}$,

$C_{n}=\lim _{t \rightarrow \infty} C_{n}\left(\tau_{0}, t\right)=A_{n} e^{-\mu \frac{\tau_{0}}{n}}$, where $A_{n}$ is determined from

$\int_{0}^{\tau_{0}} C_{n}\left(\tau_{0}, t\right) d t=n=A_{n} \frac{n}{\mu}\left(1-e^{-\mu \tau_{0} / n}\right)$

So, $A_{n}=\frac{\mu}{1-e^{-\mu \tau} 0 / n}$

Now $C_{n}=\frac{n}{\tau_{0}}, C_{n+1}=\frac{n+1}{\tau_{0}}$ and $C_{1}=\frac{1}{\tau_{0}}$

Then the steady state equations are

$0=-\lambda p_{0}+\left(\mu+\tau_{0}^{-1}\right) p_{1}$

$0=\lambda r^{-2(n-1)} p_{n-1}-\left(\lambda r^{-2 n}+\mu+C_{n}\right) p_{n}+\left(\mu+C_{n+1}\right) p_{n+1}$

From above we get

$p_{2}=\frac{\lambda^{2} r^{-2} p_{0}}{\left(\mu+\tau_{0}^{-1}\right)\left(\mu+2 \tau_{0}^{-1}\right)}$

$$
p_{1}=\frac{\lambda p_{0}}{\left(\mu+\tau_{0}^{-1}\right)}
$$

$p_{3}=\frac{\lambda^{3} r^{-6} p_{0}}{\left(\mu+\tau_{0}^{-1}\right)\left(\mu+2 \tau_{0}^{-1}\right)\left(\mu+3 \tau_{0}^{-1}\right)}$

Now inductively,

$$
\begin{aligned}
p_{n} & =\frac{\lambda^{n} r^{-(n-1) n} p_{0}}{\left(\mu+\tau_{0}^{-1}\right)\left(\mu+2 \tau_{0}^{-1}\right) \ldots\left(\mu+n \tau_{0}^{-1}\right)} \\
& =\frac{\Gamma(\sigma+1)}{\Gamma(\sigma+n+1)} \delta^{n} r^{-n(n-1)} p_{0} \quad, \text { where } \sigma=\mu \tau_{0} \text { and } \delta=\lambda \tau_{0}
\end{aligned}
$$

Finally the total probability of the system being unity, if we have no upper limit to the customer.

So $\sum_{n=0}^{\infty} p_{n}=1$

$$
\begin{aligned}
& \text { Hence } p_{0}=\left[1+\sum_{n=1}^{\infty} \frac{\Gamma(\sigma+1)}{\Gamma(\sigma+n+1)} \delta^{n} r^{-n(n-1)}\right]^{-1} \\
& =\frac{1}{H(r, \sigma, \delta)}(\text { say }) \\
& p_{n}=\frac{\frac{\Gamma(\sigma+1)}{\Gamma(\sigma+n+1)} \delta^{n} r^{-n(n-1)}}{H(r, \sigma, \delta)}
\end{aligned}
$$

Where $H(r, \sigma, \delta)=1+\sum_{n=1}^{\infty} \frac{\Gamma(\sigma+1)}{\Gamma(\sigma+n+1)} \delta^{n} r^{-n(n-1)}$

Let, $\mathrm{L}$ be the expected (average) number of units in the system.

Now $H_{\delta}=\frac{\partial H}{\partial \delta}=\sum_{n=1}^{\infty} \frac{\Gamma(\sigma+1) n \delta^{n-1} r^{-n(n-1)}}{\Gamma(\sigma+n+1)}$

$L_{s}=\sum_{n=0}^{\infty} n p_{n}$

$$
\begin{aligned}
& =\frac{\sum_{n=0}^{\infty} \frac{n \Gamma(\sigma+1) \delta^{n} r^{-n(n-1)}}{\Gamma(\sigma+n+1)}}{H(r, \sigma, \delta)} \\
& =\frac{\delta H_{\delta}(r, \rho, \delta)}{H(r, \sigma, \delta)}
\end{aligned}
$$

Let $L_{q}$ be the expected (average) queue length of this system.

$$
\begin{aligned}
L_{q} & =L_{s}-1+p_{0} \\
& =\frac{\left.\delta H_{\delta}(r, \sigma, \delta)-H(r, \sigma, \delta)+1\right]}{H(r, \rho, \delta)}
\end{aligned}
$$

\section{Conclusion}

This theory has been developed in an attempt to predict the fluctuating demands of queue length and units in the system and to enable an enterprise to provide adequate service for its customers with chi-square queues and poison balking probabilities. 


\section{Acknowledgement}

I am thankful to my guide Dr.N.N.Nayak,Prof.of Mathematics,OUAT for his valuable suggestion.

\section{References}

[1] D.Y.Barrer,Queueing with impatient customers and indifferent clerks, Operation Research,Vol.5,No.5.1957.

[2] E.Koenigsberg,Queueing with special service, Operation Research,Vol.4,213-220, 1956.

[3] R.G.Miller,A contribution to the theory of balk queues,J.Roy Statist.Soc., Vol.21,No.2,320-337,1959.

[4] G.G.O,Brien, The solution of some queuing problems,J.Soc.Ind.Appl.Math., Vol.2,133-142,1954. 